\title{
Observation of Oriented Molecular Assemblies on ITO Surfaces Using Porphyrin Derivatives Bearing Carboxyl Groups and Their Electrochemical Responses
}

\section{Katsuhiko KANAIZUKA, ${ }^{a}, *$ Shigeta YAGYU, ${ }^{a}$ Hiromi TAKAHASHI, ${ }^{b}$ Manabu ISHIZAKI, ${ }^{a}$ Masatomi SAKAMOTO, ${ }^{a}$ and Masato KURIHARA ${ }^{a}$}

\author{
a Department of Material and Biological Chemistry, Faculty of Science, Yamagata University, \\ 1-4-12 Kojirakawa-machi, Yamagata 990-8560, Japan \\ b System Instruments Co., Ltd., 776-2, Komiya-cho, Hachioji-shi, Tokyo 192-0031, Japan \\ *Corresponding author: kanaizuka@sci.kj.yamagata-u.ac.jp
}

\begin{abstract}
Control of the molecular orientation and its in situ observation have successfully been carried out. The attachment of porphyrin derivatives bearing non-, one-, and four-carboxyl groups on ITO electrode surfaces was monitored using time-resolved enhancement evanescent wave electro-absorption spectroscopy. The stability of the porphyrin self-assembled monolayers was also determined by the combination of cyclic voltammetry and evanescent wave electronic spectroscopy measurements.
\end{abstract}

(C) The Electrochemical Society of Japan, All rights reserved.

Keywords : Porphyrin, Molecular Orientation, Evanescence Wave, Redox

\section{Introduction}

The molecular orientation on electrode surfaces has been the key subject for the development of molecular devices, such as highperformance solar cells, memory devices, light emission diodes, catalytic devices, and chemical sensors. ${ }^{1-8}$ From these viewpoints, many efforts have been devoted to control the orientation of monolayers and multilayers on electrodes. ${ }^{9-13}$ For example, Matsuo and Nakamura reported that the photocurrent direction can be switched by changing the orientation of a fullerene derivative on an electrode. ${ }^{10}$ The clarification of the orientation of such functional molecules often has been carried out using X-ray diffraction measurements. ${ }^{11-13}$ Nevertheless, it is generally difficult to conveniently determine the molecular orientation on electrode surfaces.

A porphyrin derivative is one of the most available molecules to understand the molecular orientation and electrochemical properties on an electrode surface because of their flat and rigid chemical structures $^{14,15}$ and their unique redox and photophysical properties. ${ }^{16}$ Porphyrins are well-known for their photo-responsive and redoxactive molecules; therefore, porphyrins are used for the construction of photocurrent generating systems. ${ }^{17-21}$ Coin-shaped molecules, such as porphyrins and phthalocyanine, show different absorption spectra to the direction of light. ${ }^{22,23}$ We have tried to establish a convenient system in order to understand the orientation of porphyrins on an electrode and their electrochemical behavior.

A molecule bearing carboxyl or phosphonic acid groups can be chemically fixed on an indium-tin oxide (ITO) electrode and form a thermodynamically stable film, a so-called self-assembled monolayer (SAM). ${ }^{10,24-26}$ At this time, we introduced some carboxyl groups onto the porphyrin backbone to fix and control the orientation of the porphyrin derivatives at substrates. Scheme 1a is an image of a porphyrin derivative perpendicularly fixed on a substrate; on the other hand, Scheme $1 \mathrm{~b}$ shows molecular immobilization parallel to the substrate.

\section{Experimental}

Three molecules were employed to understand the molecular
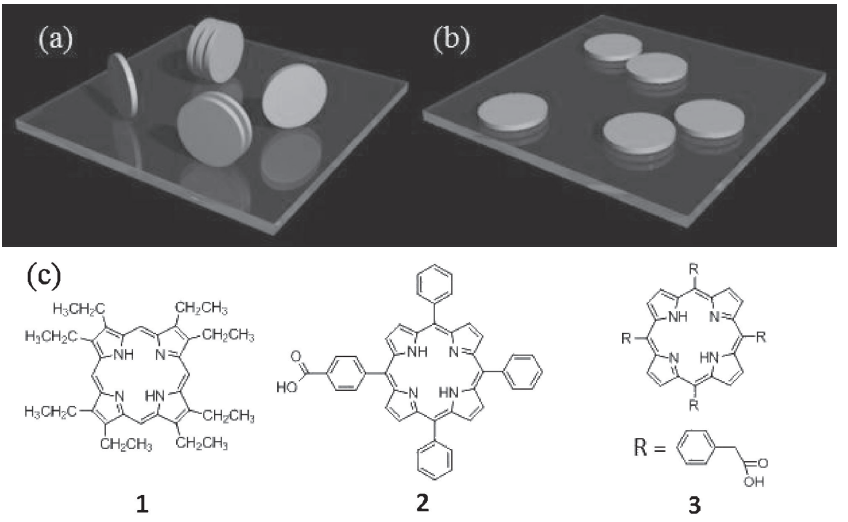

Scheme 1. Images of molecular fixation perpendicular (a) and parallel (b) to the substrate, where gray coins are presented by porphyrin derivatives. Chemical structures of porphyrin derivatives used in this study (c).

orientations on electrodes, 2,3,7,8,12,13,17,18-octaethyl-21H,23Hporphyrin, 1; 5-(4-carboxyphenyl)-10,15,20-triphenylporphyrin, 2; 5,10,15,20-tetrakis(4-carboxymethyloxyphenyl)porphyrin, 3 (Scheme 1c). LaSFn1 (Sumita Optical Glass, INC) high refractive index 1.80 glass was used as an optical waveguide $(0.4 \mathrm{~mm}$ thickness $20 \mathrm{~mm} \times 65 \mathrm{~mm})$. This waveguide was coated ITO $(30 \mathrm{~nm}$ thickness $20 \mathrm{~mm} \times 20 \mathrm{~mm}, 100 \Omega / \mathrm{cm})$ as the working electrode, platinum $(\mathrm{Pt})$ wire as the counter electrode and a polished silver (Ag) wire electrode as the reference electrode. The substrate was immersed in $0.1 \mathrm{mmol} / \mathrm{L}$ chloroform or DMF solutions of porphyrin derivatives, 1-3, and, at the same time, time-resolved enhancement evanescent wave electro-absorption spectroscopy (TRES) was carried out using an optical waveguide system (Surface and Interface spectrometer SIS-5000, System Instruments CO., LTD., Japan). The ITO was then thoroughly washed with chloroform (for compounds 1 and 2) or DMF (for compound 3) in order to remove physically adsorbed porphyrin derivatives and contaminants. A 
cyclic voltammetry measurement was carried out in a $0.1 \mathrm{~mol} / \mathrm{L}$ $\mathrm{KCl}$ aqueous solution at the scan rate of $0.1 \mathrm{~V} / \mathrm{s}$ using CHI611 A electrochemical analyzer (CH Instruments, USA), and, at the same time, TRES was performed by optical waveguide system, and the spectra were recorded at $5 \mathrm{~s}$ intervals. A Xe150W lamp was used as the light source.

\section{Results and Discussion}

The time-dependence absorption spectra of ITO in the solutions of $\mathbf{2}$ and $\mathbf{3}$ are shown in Figs. $1 \mathrm{a}$ and $1 \mathrm{~b}$, respectively. The absorbance of ITO was gradually increased in both systems and saturated within a few hours. A clear electronic spectrum was not obtained when an ITO was immersed in the solution of $\mathbf{1}$, probably because 1 had no substitution group on the porphyrin backbone for molecular fixation. This indicates the carboxyl group acted as an adsorbent for the ITO surface. The intensity of the Q band of $\mathbf{3} /$ ITO (hereafter, / denotes an interface) was observed to be stronger than that of 2/ITO. The ratios of the peak top of Soret/Q bands $(512 \mathrm{~nm})$ were estimated to be ca. 35 and 18 for $2 /$ ITO and $3 /$ ITO, respectively (comparing $75 \mathrm{~min}$ in Fig. 1a with $135 \mathrm{~min}$ in $1 \mathrm{~b}$ ). The surface coverage of the compounds $\mathbf{2}$ and $\mathbf{3}$ on flat ITO electrodes (Fruuchi Chemical Corporation $10 \mathrm{ohms} / \mathrm{sq}$ ) were estimated from the areas of anodic current to be 13.5 and $9.8 \times 10^{-11} \mathrm{~mol} / \mathrm{cm}^{2}$, respectively (the immersion period of time was $24 \mathrm{~h}$ ). In the enhancement evanescent wave electro-absorption spectroscopy, the direction of induced light was 70.5 degrees normal to the waveguide surface (see Fig. 1g). The difference of the ratio in the spectra indicates the different orientation of porphyrin on the ITO. Most of 2 was perpendicularly fixed to the ITO. On the other hand, most of $\mathbf{3}$ was perpendicularly fixed to the ITO in the initial step (the ratio of the Soret/Q bands was estimated to be 35 immersed for $15 \mathrm{~min}$ ). However, 3 was gradually moved at the ITO, and orientation changed from a perpendicular to a parallel conformation. The interaction between a coin-shaped molecule (such as phthalocyanine derivatives) and light has been well studied especially in the system of Langmuir-Blodgett films. ${ }^{27}$ When the direction of transition moment in a molecule is perpendicular to that of light, the intensity of absorption can be weakly observed.

Figures $1 \mathrm{c}$ and $1 \mathrm{e}$ show the absorption spectra of $2 /$ ITO at $0 \mathrm{~V}$ vs. Ag after applied potentials in a $0.1 \mathrm{~mol} / \mathrm{L} \mathrm{KCl}$ aqueous solution. In Fig. 1c, a bias was applied between +0.8 and $-0.8 \mathrm{~V}$ at a scan rate of $0.1 \mathrm{~V} / \mathrm{s}$. The cyclic voltammograms $(\mathrm{CVs})$ are shown in Fig. 1d. No spectral change was observed in this range, which indicated that molecules on the ITO were not damaged at all. On the other hand, absorbance in electronic spectra gradually decreased after scanning at the biases between +1.2 and $-1.2 \mathrm{~V}$ (Fig. 1e). In the CVs (Fig. 1f), an irreversible redox was observed. The molecule of 2-fixed ITOs was oxidized at the potential of $0.8 \mathrm{~V}\left(E_{\mathrm{pa}}\right.$ was estimated to be $1.18 \mathrm{~V})$. Porphyrin is known to show the reversible redox events in a solution (compound 2 shows a reversible redox at 0.86 and $-1.9 \mathrm{~V}$ vs. $\mathrm{Ag} / \mathrm{Ag}^{+}$in $\mathrm{CH}_{2} \mathrm{Cl}_{2}$ ); thus, this decrease of the absorbance indicates that the fixed molecules were removed from the ITO surface probably because of electric repulsion of the oxidized molecule and the electrode. We have previously reported these removal behaviors of functional molecules from electrodes. ${ }^{10}$ 3/ITO also showed a very similar redox event and absorption spectral change to $2 /$ ITO. The difference between $2 /$ ITO and $3 /$ ITO was the decrease speed of absorbance upon applied bias to the film. This decrease speed in $3 /$ ITO was higher than $2 /$ ITO because the distance between the porphyrin backbone and the ITO was closer in the case of $\mathbf{3} /$ ITO. Unfortunately, this time we could not clarify the rate of the removal and conformational change upon applied voltage of the compounds on the electrode. The molecule of 3 may form perpendicular the electrode in a short period of time upon oxidation.
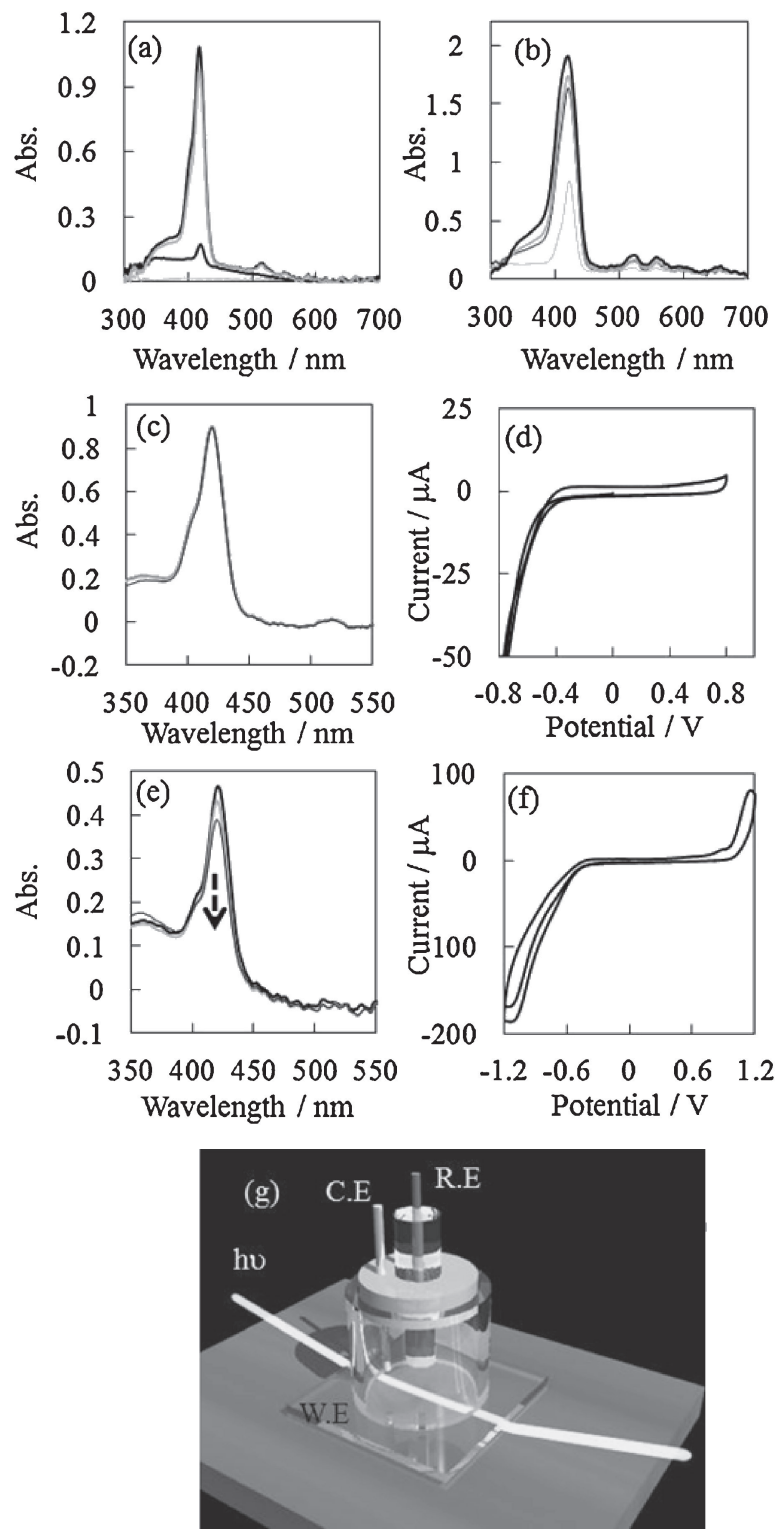

Figure 1. Time-resolved enhancement evanescent wave electroabsorption spectra (TRES) of ITO in the solutions of $\mathbf{2}$ (gray: 0, black: 15, gray bold: 60, and black bold: $75 \mathrm{~min}$ ) (a), $\mathbf{3}$ (gray: 15 , black: 30, gray bold: 45 , and black bold: $135 \mathrm{~min}$ ) (b), (c) TRES of 2/ITO under the applied bias at $0 \mathrm{~V}$ (black bold), after scan between +0.8 and $-0.8 \mathrm{~V}$ vs. Ag [gray bold (after 2 cycles), and black (after 3 cycles)], (d) cyclic voltammograms (CVs) of $2 /$ ITO, (e) TRES of $2 / \mathrm{ITO}$ at $0 \mathrm{~V}$ after the applied bias between +1.2 and $-1.2 \mathrm{~V}$, and (f) CVs of 2/ITO. (g) Image of the cell in order to determine the stability of the porphyrin SAM by the combination of cyclic voltammetry and evanescent wave electronic spectroscopy measurements.

\section{Conclusion}

The molecular orientation and its electrochemical behavior were successfully and conveniently examined by the combination of cyclic voltammetry and evanescent wave electronic spectroscopy measurements. The orientation of a porphyrin bearing one carboxyl group was perpendicular to the ITO electrode. On the other hand, the porphyrin bearing four carboxyl groups was initially fixed perpendicularly to the ITO. This orientation was gradually changed and became parallel to the ITO. The orientation of the porphyrin derivatives was determined by the ratio of the $\mathrm{Q}$ and Soret bands. 
We developed a method for the determination of the molecular orientation at the electrode surfaces. Furthermore, the oxidized or reduced properties of the functional molecular film could be clarified. In order to develop the molecular devices such as organic FET, EL, and solar cells, speedy understanding of the molecular orientation at electrode surfaces is necessary. Our new approach is an effective method for the preparation and understanding of molecule-based electronic devices.

\section{Acknowledgment}

This research was supported by Dissemination of Tenure Tracking System Program of Ministry of Education, Culture, Sports, Science and Technology-Japan. This work was also partially supported by KAKENHI (No. 22750059).

\section{References}

1. J. V. Barth, G. Costantini, and K. Kern, Nature, 437, 671 (2005).

2. S. Yasutomi, T. Morita, Y. Imanishi, and S. Kimura, Science, 304, 1944 (2004).

3. C. Lin and C. R. Kagan, J. Am. Chem. Soc., 125, 336 (2003).

4. L. Motiei, M. Lahav, D. Freeman, and M. E. Boom, J. Am. Chem. Soc., 131, 3468 (2009).

5. L. Motiei, Y. Yao, J. Choudhury, H. Yan, T. J. Marks, M. E. Boom, and A. Facchetti, J. Am. Chem. Soc., 132, 12528 (2010).

6. A. Yella, H.-W. Lee, H. N. Tsao, C. Yi, A. K. Chandriran, M. K. Nazeeruddin, E. W.-G. Diau, C.-Y. Yeh, S. M. Zakeeruddin, and M. Grätzel, Science, 334, 629 (2011).

7. F. B. Abdelrazzaq, R. C. Kwong, and M. E. Thompson, J. Am. Chem. Soc., 124 4796 (2002).

8. M. M. Richter, Chem. Rev., 104, 3003 (2004).
9. K. Terada, K. Kanaizuka, V. M. Iyer, M. Sannodo, S. Saito, K. Kobayashi, and M. Haga, Angew. Chem., Int. Ed., 50, 6287 (2011).

10. Y. Matsuo, K. Kanaizuka, K. Matsuo, Y.-W. Zhong, T. Nakae, and E. Nakamura, J. Am. Chem. Soc., 130, 5016 (2008).

11. K. Kanaizuka, R. Haruki, O. Sakata, M. Yoshimoto, Y. Akita, and H. Kitagawa, J. Am. Chem. Soc., 130, 15778 (2008).

12. D. Wang, J. Liu, Q. Huo, Z. Nie, W. Lu, R. E. Williford, and Y.-B. Jiang, J. Am. Chem. Soc., 128, 13670 (2006).

13. M. Altman, A. D. Shukla, T. Zubkov, G. Evmenenko, P. Dutta, and M. E. Boom, J. Am. Chem. Soc., 128, 7374 (2006).

14. J. Otsuki, E. Seki, T. Taguchi, M. Asakawa, and K. Miyake, Chem. Lett., 36, 740 (2007).

15. R. Makiura, S. Motoyama, Y. Umemura, H. Yamanaka, O. Sakata, and H. Kitagawa, Nat. Mater, 9, 565 (2010)

16. For example, S. Yoshimoto, E. Tsutsumi, K. Suto, Y. Honda, and K. Iataya, Chem. Phys., 319, 147 (2005).

17. D.-C. Lee, G. M. Morales, Y. Lee, and L. Yu, Chem. Commun., 100 (2006).

18. K. E. Splan and J. T. Hupp, Langmuir, 20, 10560 (2004).

19. K. Uosaki, T. Kondo, X.-Q. Zhang, and M. Yanagida, J. Am. Chem. Soc., 119, 8367 (1997)

20. K. Ikeda, K. Takahashi, T. Masuda, and K. Uosaki, Angew. Chem., Int. Ed., 50, 1280 (2011)

21. H. Imahori, H. Norieda, H. Yamada, Y. Nishimura, I. Yamazaki, Y. Sakata, and S. Fukuzumi, J. Am. Chem. Soc., 123, 100 (2001).

22. F. Bonosi, G. Ricciardi, and F. Lelj, Thin Solid Films, 243, 310 (1994).

23. C.-F. Lo, L. Luo, E. W.-G. Diau, I.-J. Chang, and C.-Y. Lin, Chem. Commun., 1430 (2006).

24. A. Ulman, An Introduction to Ultrathin Organic Films from Langmuir-Blodgett to Self-Assembly, Academic Press, San Diego (1991).

25. B. Long, K. Nikitin, and D. Fitzmaurice, J. Am. Chem. Soc, 125, 5152 (2003).

26. M. Curreli, C. Li, Y. Sun, B. Lei, M. A. Gundersen, M. E. Thompson, and C. Zhou, J. Am. Chem. Soc., 127, 6922 (2005).

27. For example, K. Lewandowska, D. Wrobel, A. Biadasz, and R. Swietlik, J. Photochem. Photobiol., A, 200, 225 (2008). 